\title{
Clinical characteristics in Japanese children with nonobese type 2 diabetes
}

Tatsuhiko Urakami, MD, PhD

Department of Pediatrics, Nihon University School of Medicine, Tokyo, Japan
Received: 12 August, 2018

Revised: 4 September, 2018

Accepted: 5 September, 2018

Address for correspondence:

Tatsuhiko Urakami, MD, PhD

Department of Pediatrics, Nihon

University School of Medicine, 1-6

Kandasurugadai, Chiyodaku Tokyo

101-8309, Japan

Tel: +81-3-32931711

Fax: +81-3-3292-2880

E-mail: urakami.tatsuhiko@nihon-u. ac.jp

https://orcid.org/0000-0001-79769557
It is well known that the incidence of youth-onset type 2 diabetes is increasing worldwide. On the other hand, most studies have shown that the majority of youthonset type 2 diabetes occurs in obese individuals, generally with a body mass index (BMI) greater than the 90th or 95th centile for sex- and age-matched children and adolescents. However, we identified some Japanese children with nonobese type 2 diabetes and BMI less than 90th centile by a urine glucose screening program at schools in the Tokyo Metropolitan Area. According to the results obtained from the screening, clinical characteristics of patients with nonobese type 2 diabetes seemed to be different from those in obese type 2 diabetes. Nonobese patients tended to show lower insulin secretion abilities and milder, but evident, insulin resistance from the time of diagnosis. Female, low birth weight (small for gestational age), and genetic background, not related to $\beta$-cell-associated autoimmunity, may play a role in development of nonobese type 2 diabetes. In addition, nonobese patients tend to progress earlier to pharmacological treatment including oral hypoglycemic drugs and insulin. Further studies are needed to confirm to these findings and clarify the pathophysiology of children with nonobese type 2 diabetes.

Keywords: Type 2 diabetes, Child, Nonobese, Insulin resistance, Insulin secretion, Pharmacological treatment

\section{Introduction}

It is well known that the incidence of youth-onset type 2 diabetes is increasing worldwide. ${ }^{1)}$ The SEARCH for Diabetes in Youth population-based study (SEARCH Study) recently reported on estimated trends in the incidence of type 1 and type 2 diabetes among young people at 5 centers in the USA during 2002-2012. ${ }^{2)}$ According to the report, the overall incidence of youth-onset type 2 diabetes has increased by $7.1 \%$ annually (from $9.0 / 100,000 / \mathrm{yr}$ during 2002-2003 to 12.5/100,000/yr during 2011-2012, $P<0.001$ ). Other study performed in 14 medical centres in China found that the incidence of youth-onset type 2 diabetes increased from 4.1/100,000/yr during October 1995-September 2000 to 10.0/100,000/yr during October 2005-September 2010. Furthermore, the total number of type 2 diabetes increased 4 -fold during this period. ${ }^{3)}$ On the other hand, youth-onset type 2 diabetes occurs in all ethnic groups, however, there exists a considerable difference in the incidence among races. ${ }^{1,2,4)}$ Nonwhite Caucasian descent, such as Black African descent, native North American, HispanicAmerican and Asian, have a much greater prevalence than White-Caucasian descent. Asians are recognized as one of the highest prevalence of youth-onset type 2 diabetes among ethnic groups, as findings in Hong Kong (90\% of youth-onset diabetes was type 2 diabetes) and Taiwan (50\%) have shown. ${ }^{1)}$ We previously demonstrated a high incidence of type 2 diabetes using a unique school-based screening program (urine glucose screening at schools, Fig. 1). ${ }^{5-}$ ${ }^{8)}$ We found that the incidence was 2.5-3.5/100,000 students/year, which was estimated to be nearly twice that reported for type 1 diabetes in Japanese children. ${ }^{7)}$ According to the study 


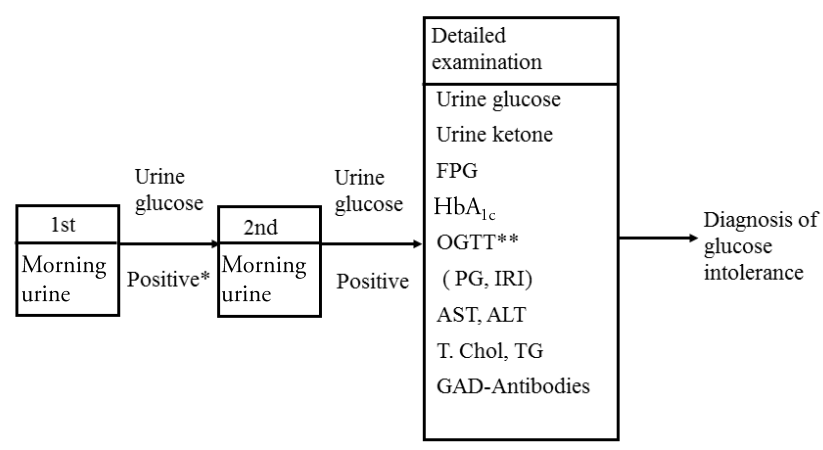

Fig. 1. Urine glucose screening program at schools in the Tokyo Metropolitan Area. FPG, fasting plasma glucose; $\mathrm{HbA}_{1 c}$ glycated hemoglobin; OGTT, oral glucose tolerance test; PG, plasma glucose; IRI, immunoreactive insulin; AST, aspartate aminotransferase; ALT, alanine aminotransferase; T. Chol, total cholesterol; TG, triglyceride; GAD-Antibodies, antibodies against glutamic acid decarboxylase. ${ }^{*}$ Urine glucose $\geq 100 \mathrm{mg} / \mathrm{dL}$. ${ }^{*} \mathrm{OGTT}, 1.75 \mathrm{~g} / \mathrm{kg}$ of glucose, maximum $75 \mathrm{~g}$.

of a urine glucose screening program at schools in the Tokyo Metropolitan Area during 1974-2010, the overall incidence of type 2 diabetes was 2.66/100,000 students/year. Junior high school students had a significantly higher incidence than primary school students (0.85 vs. $6.47 / 100,000$ students/yr, $P<0.0001)^{8)}$

On the other hand, most studies have shown that the majority of youth-onset type 2 diabetes is obese, generally with the body mass index (BMI) more than the 90th or 95th percentile for sex- and age-matched children and adolescents. However, we found some patients with nonobese type 2 diabetes, with the BMI less than 90th percentile for Japanese children, by a urine glucose screening program at schools conducted in the Tokyo Metropolitan Area. They have different clinical features from obese type 2 diabetes. We therefore reviewed the clinical characteristics of Japanese children with nonobese type 2 diabetes detected by the screening program at schools.

\section{Clinical characteristics in Japanese children with nonobese type 2 diabetes}

\section{Urine glucose screening program at schools in the Tokyo Metropolitan Area}

We have annually conducted a urine glucose screening towards primary school children, aged 7-12 years, and junior high school children, aged 13-15 years, residing in the Tokyo Metropolitan Area. ${ }^{5,6)}$ During the period from 1975 to 2015, a total of $11,652,205$ students, including $7,955,857$ primary school students and 3,696,348 junior high school students, participated in the urine glucose screening program. Finally, a total of 301 students, including 64 primary school students and 237 junior high school students, were diagnosed as having type 2 diabetes. The incidence of type 2 diabetes (per 100,000 students/yr) during the entire study period was 2.58 overall with
0.80 in primary school students and 6.41 in junior high school students."

Type of diabetes was retrospectively determined to follow up the clinical course for at least 1 year after diagnosis. Subjects diagnosed with type 2 diabetes usually sustained considerable insulin secretion abilities and did not require insulin treatment, and continued adequate glycemic control under dietary management, physical exercise or by using some oral hypoglycemic drugs within at least 3 years after diagnosis. Then, some patients subsequently progressed to insulin treatment, use of basal insulin or premixed insulin, to achieve optimal glycemic control. Besides, all the patients had no evidence of autoimmunity to pancreatic $\beta$-cells, without showing positive results in any $\beta$-cell associated autoantibodies. ${ }^{5-7)}$

We previously reported that a small number of school children with slowly progressive form of type 1 diabetes were also detected by the screening program. ${ }^{5,10,11)}$ In Japan, there is clinical heterogeneity of youth-onset type 1 diabetes, including abrupt-onset type 1 diabetes, and slowly progressive type 1 diabetes. The latter type exhibits a milder onset of the disease without showing ketoacidosis at the time of diagnosis. This novel type is generally encountered in adults and has been referred to as latent autoimmune diabetes in adults. ${ }^{12,13)}$ Patients with slowly progressive type 1 diabetes require insulin treatment at least within 1.5 to 3 years from the diagnosis, and most of them have positive $\beta$-cell associated autoantibodies. ${ }^{5,10,11)} \mathrm{On}$ the other hand, patients diagnosed with type 2 diabetes have no evidence of $\beta$-cell associated autoimmunity.

Monogenic diabetes, such as mitochondrial diabetes and maturity-onset diabetes in the young (MODY), can be detected by the screening program. ${ }^{9)}$ They are tested for mitochondrial DNA mutations and MODY related genes, and are eventually identified as monogenic diabetes.

\section{Frequency and clinical features including age, sex, and family history in nonobese type 2 diabetes}

Among the 301 students diagnosed with type 2 diabetes by the screening program during 1975-2015, 39 (13.0\%) were identified as nonobese type 2 diabetes, with the BMI less than the 90th percentile for sex- and age-matched Japanese children." Age at the diagnosis was $12.2 \pm 2.0$ years, which was not different from that in obese-patients ( $12.8 \pm 3.0$ years). On the other hand, of note, the percentage of females was significantly greater among the non-obese patients than the obese-patients (84.6\% vs. $49.2 \%, P<0.005)$. This finding suggests that femaleness might play a role in developing subtype of type 2 diabetes. Besides, a positive family history in the first- and second-degree relatives was in $61.5 \%$ of the nonobese patients, which was not different from that in obese-patients $(65.6 \%)$. Interestingly, $75.0 \%$ of the relatives of the nonobese patients had a similar diabetic type; i.e., nonobese type 2 diabetes. Therefore, there might be a strong hereditary component, likely a multigenetic determinant, in the development of nonobese type 2 diabetes. 


\section{Birth weight in nonobese type 2 diabetes}

We did not analyze the data regarding birth weight in patients diagnosed with type 2 diabetes by the screening program during 2009-2015. Besides, the data from 1975 to 2008 was analyzed, and we found a significant high frequency of small for gestational age (SGA) in non-obese patients. ${ }^{14)}$ The mean birth weight of the nonobese patients was not different from that of obese-patients $(2,865 \pm 480 \mathrm{~g}$ vs. $3,002 \pm 250 \mathrm{~g})$. On the other hand, frequencies of SGA and large for gestational age in nonobese patients were $20.8 \%$ and $8.3 \%$, respectively, and those in obese patients were $8.5 \%$ and $10.3 \%$, respectively. The percentage of SGA of the nonobese patients was much greater than that recorded in the Japanese general population $(6.3 \%$, $P<0.0001)$ (Fig. 2). Sugihara et al. ${ }^{15)}$ also reported that the frequencies of both low and high birth weights were higher in patients with type 2 diabetes than in Japanese control subjects. These findings suggest possibility of an association between intrauterine growth failure and the risk of type 2 diabetes, particularly in nonobese type 2 diabetes.

\section{Laboratory data at diagnosis in nonobese type 2 diabetes}

The data from 1974 to 2008 also showed comparison of laboratory data at the time of diagnosis between nonobese patients and obese-patients as indicated Table 1 and Fig. $3 .{ }^{14)}$ There was no significant difference in the mean levels of fasting plasma glucose and glycosylated hemoglobin, expressed as the National Standardization Program value, between the two groups. However, nonobese patients had significantly lower mean values of fasting plasma immunoreactive insulin (IRI) and homeostasis model assessment of insulin resistance than obese patients. Moreover, they also showed significantly decreased

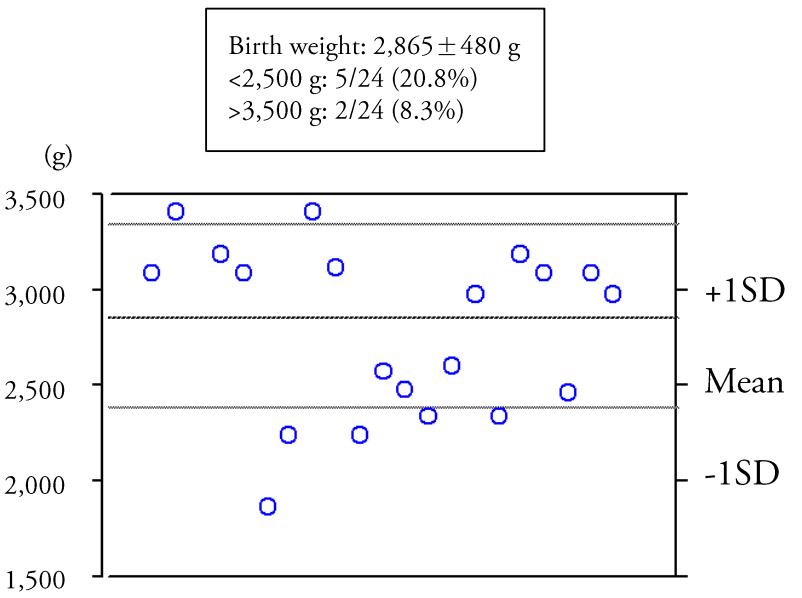

Fig. 2. Birth weight in children with nonobese type 2 diabetes. SD, standard deviation. mean value of homeostasis model assessment of $\beta$-cell function than obese patients, and impaired first phase insulin secretion response, expressed as insulinogenic index (calculated by $\Delta I R I$ (30 minutes on OGTT)/APG (30 minutes on OGTT), of less than 0.4. However, we also found that nonobese patients showed significantly higher insulin resistance as compared with ageand pubertal stage-matched Japanese people without diabetes in other studies (Fig. 4). ${ }^{16,17)}$ Accordingly, nonobese patients show lower insulin secretion abilities, but have milder, but evident, insulin resistance even from the time of diagnosis.

\section{Accumulation of visceral adiposity-possible cause in nonobese type 2 diabetes}

Yoon et al. ${ }^{18)}$ reported that even though the average BMI of Asians was lower than that in White Caucasian descents, the prevalence of type 2 diabetes was higher in Asians, possibly due to more accumulation of visceral adiposity. Some previous studies also discussed that Asians might have a greater amount of visceral adiposity than Caucasians. ${ }^{1920)}$ Then, we examined the ratio of a visceral fat area to a subcutaneous fat area in some patients with obese and nonobese type 2 diabetes. It is notable that some nonobese patients have a large amount of visceral fat areas, even though their body weights and subcutaneous

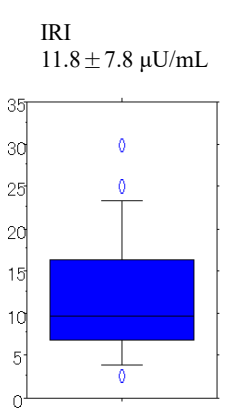

$\operatorname{IRI}(\mu \mathrm{U} / \mathrm{mL})$

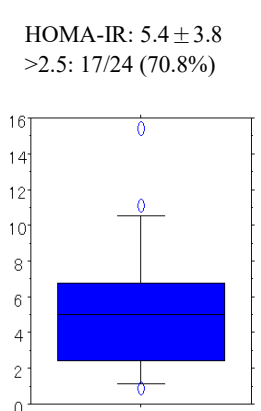

HOMA-IR

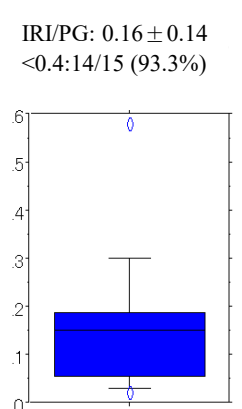

IRI/PG
Fig. 3. Laboratory data at diagnosis in children with nonobese type 2 diabetes. IRI, immunoreactive insulin; HOMA-IR, homeostasis model assessment of insulin resistance; $\mathrm{PG}$, plasma glucose.

Table 1. Laboratory data of nonobese and obese patients with type 2 diabetes at the time of diagnosis

\begin{tabular}{lccc}
\hline Variable & Nonobese patients & Obese patients & $P$-value \\
\hline $\mathrm{FPG}(\mathrm{mg} / \mathrm{dL})$ & $133.5 \pm 86.5$ & $148.5 \pm 28.0$ & $\mathrm{NS}$ \\
$\mathrm{HbA}_{1 \mathrm{c}}(\%)$ & $8.4 \pm 4.2$ & $8.6 \pm 1.1$ & $\mathrm{NS}$ \\
Fasting IRI $(\mu \mathrm{U} / \mathrm{mL})$ & $11.8 \pm 7.8$ & $30.0 \pm 18.7$ & $<0.001$ \\
HOMA-IR & $5.4 \pm 3.8$ & $13.3 \pm 8.0$ & $<0.005$ \\
HOMA- $\beta$ & $96.1 \pm 55.0$ & $162.3 \pm 107.4$ & $<0.001$ \\
Insulinogenic index & $0.16 \pm 0.14$ & $\mathrm{ND}$ & -
\end{tabular}

Values are presented as mean \pm standard deviation.

FPG, fasting plasma glucose; $\mathrm{HbA}_{1 c}$, glycosylated hemoglobin; IRI, immunoreactive insulin; HOMA-IR, homeostasis model assessment of insulin resistance; HOMA- $\beta$, homeostasis model assessment of $\beta$-cell function; NS, not significant; ND, no data. 
fat areas reveal normal for their age and sex, and show higher ratio of a visceral fat area to a subcutaneous fat area (Fig. 5). Therefore, it is necessary to evaluate the amounts of visceral adiposity, other than subcutaneous adiposity, which could more precisely reflect insulin resistance in patients with nonobese type 2 diabetes.

\section{Treatment option}

Dietary management and physical exercise are principal in treatment of type 2 diabetes. Most patients with obese-type 2 diabetes can improve glycemic control by reducing their body weight. ${ }^{8,21,22)}$ However, some patients fail to improve glycemic control even though therapeutic modification of the diet and exercise habits. Particularly, patients with nonobese type 2 diabetes tend to progress earlier to pharmacological treatment using oral hypoglycemic agents (OHA) or insulin. We found over $95 \%$ of patients with nonobese type 2 diabetes eventually progressed to pharmacological treatment: i.e., $45.8 \%$ treated with OHA and $50.0 \%$ with insulin. ${ }^{14)}$ The mean interval to start of pharmacological treatment was $3.1 \pm 2.3$ years $(2.2 \pm 2.1$ years for $\mathrm{OHD}$ and $4.8 \pm 2.9$ years for insulin). Fig. 6 shows various kinds of pharmacological treatment used in patients with obese and nonobese type 2 diabetes. Metformin should be a first-line drug, particularly in obese patients. Sulfonyl urea (SU), glimepiride, is used in mostly nonobese patients. Alpha-glucosidase inhibitors, dipeptidyl peptidase- 4 inhibitors and glucagon-like peptide- 1 receptor agonists come to be used in both types of diabetes. New drugs, SGLT2 inhibitors hereafter may be used mainly among obese patients. ${ }^{22)}$ However, only metformin and glimepiride
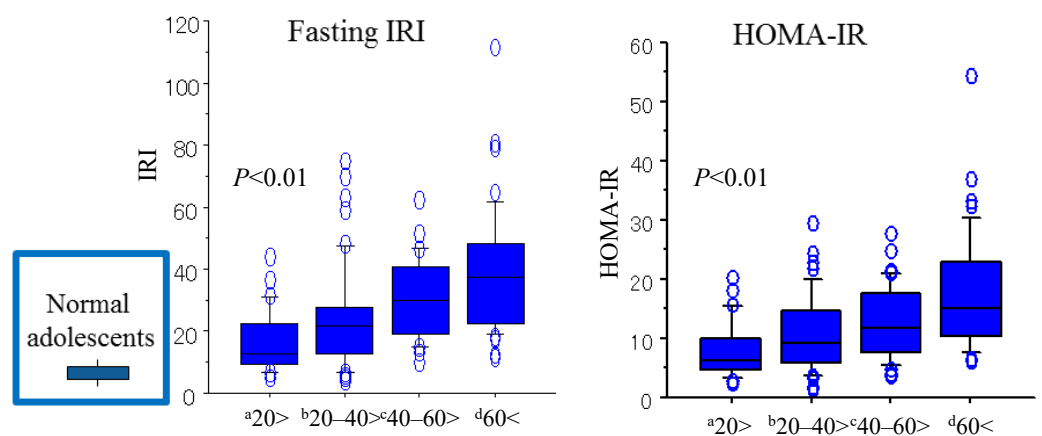

Fig. 4. Comparison of fasting IRI levels and HOMA-IR at diagnosis among children with type 2 diabetes of different \% overweight. IRI, immunoreactive insulin; HOMA-IR, homeostasis model assessment of insulin resistance. \% overweight: ${ }^{a}$ Nonobesity: $n=17,<20 \%$; Mild obesity: $n=37^{b}, n=30^{c}$; d Severe obesity: $n=26$.

Obese

14 years old, female

WC: $83.0 \mathrm{~cm}$

BMI $27.5 \mathrm{~kg} / \mathrm{m}^{2}$

SF $237.0 \mathrm{~m}^{2}$

VF $66.6 \mathrm{~m}^{2}$

VF/SF 0.28

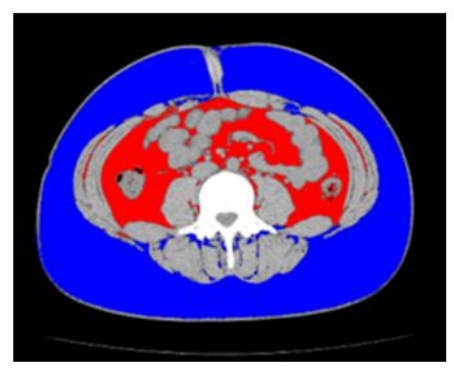

Nonobese

17 years old, female

WC: $80.6 \mathrm{~cm}$

BMI $23.4 \mathrm{~kg} / \mathrm{m}^{2}$

SF $95.8 \mathrm{~m}^{2}$

VF $117.0 \mathrm{~m}^{2}$

$\mathrm{VF} / \mathrm{SF} 1.22$

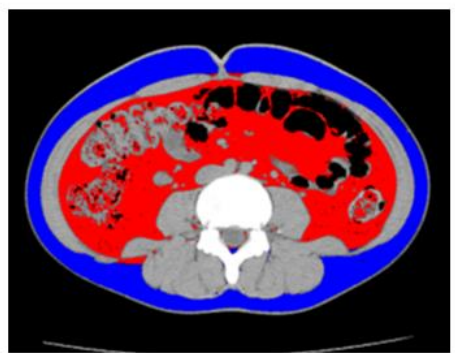

Fig. 5. Distribution of subcutaneous fat (SF) and visceral fat (VF) in patient with nonobese type 2 diabetes. WC, waist circumference; BMI, body mass index; SF, subcutaneous fat; VF, visceral fat. 

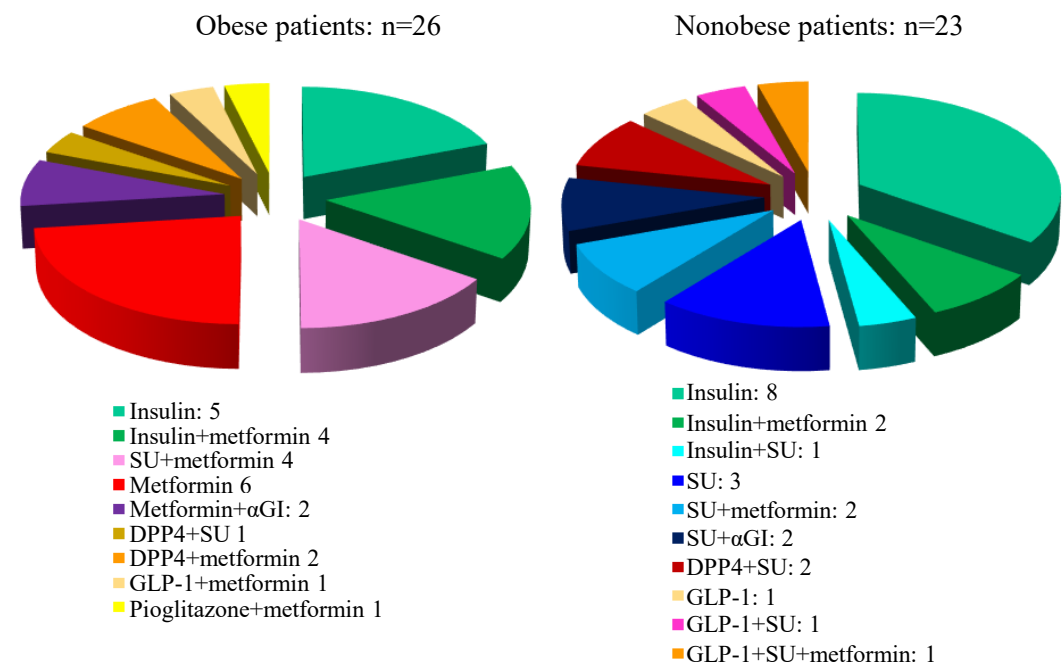

Fig. 6. Pharmacological treatment in patients with nonobese and obese type 2 diabetes. SU, sulfonyl urea; a-Gl, a-glucosidase inhibitors; DPP-4, dipeptidyl peptidase-4 inhibitors; GLP-1, inhibitors and glucagon-like peptide-1 receptor agonists.

are currently permitted for official use in pediatric patients. Therefore, it is necessary to get informed consent from the patients and their family members and be careful for occurrence of the adverse events when use of new medications other than metformin and glimepiride.

\section{Conclusions}

Clinical characteristics of nonobese type 2 diabetes seemed to be different from those in obese type 2 diabetes. Children with nonobese type 2 diabetes tend to show lower insulin secretion abilities and milder, but evident, insulin resistance even from the time of diagnosis. Femaleness, low birth weight (SGA) and some genetic background, no evidence of $\beta$-cell associated autoimmunity, may play a possible role in the development of nonobese type 2 diabetes. In addition, they tend to progress earlier to pharmacological treatment. Further studies are needed to confirm to clarify these findings.

\section{Conflict of interest}

No potential conflict of interest relevant to this article was reported.

\section{References}

1. Zeitler P, Fu J, Tandon N, Nadeau K, Urakami T, Barrett T, et al. ISPAD Clinical Practice Consensus Guidelines 2014. Type 2 diabetes in the child and adolescent. Pediatr Diabetes 2014;15 Suppl 20:26-46.

2. Mayer-Davis EJ, Lawrence JM, Dabelea D, Divers J, Isom S, Dolan L, et al. Incidence trends of type 1 and type 2 diabetes among youths, 2002-2012. N Engl J Med 2017;376:1419-29.

3. Fu JF, Liang L, Gong CX, Xiong F, Luo FH, Liu GL, et al. Status and trends of diabetes in Chinese children: analysis of data from 14 medical centers. World J Pediatr 2013;9:127-34.

4. Dabelea D, Mayer-Davis EJ, Saydah S, Imperatore G, Linder B, Divers J, et al. Prevalence of type 1 and type 2 diabetes among children and adolescents from 2001 to 2009. JAMA 2014;311:1778-86.

5. Urakami T, Miyamoto Y, Fujita H, Kitagawa T. Type 1 (insulin-dependent) diabetes in Japanese children is not a uniform disease. Diabetologia 1989;32:312-5.

6. Urakami T, Kubota S, Nitadori Y, Harada K, Owada M, Kitagawa T. Annual incidence and clinical characteristics of type 2 diabetes in children as detected by urine glucose screening in the Tokyo metropolitan area. Diabetes Care 2005;28:1876-81.

7. Urakami T, Morimoto S, Nitadori Y, Harada K, Owada M, Kitagawa T. Urine glucose screening program at schools in Japan to detect children with diabetes and its outcomeincidence and clinical characteristics of childhood type 2 diabetes in Japan. Pediatr Res 2007;61:141-5.

8. Urakami T, Suzuki J, Mugishima H, Amemiya S, Sugihara S, Kawamura T, et al. Screening and treatment of childhood type 1 and type 2 diabetes mellitus in Japan. Pediatr Endocrinol Rev 2012;10 Suppl 1:51-61.

9. Urakami T, Miyata M, Yoshida K, Mine Y, Kuwabara R, Aoki $\mathrm{M}$, et al. Changes in annual incidence of school children with type 2 diabetes in the Tokyo Metropolitan Area during 1975-2015. Pediatr Diabetes 2018.

10. Urakami T, Miyamoto Y, Matsunaga H, Owada M, Kitagawa T. Serial changes in the prevalence of islet cell antibodies and islet cell antibody titer in children with IDDM of abrupt or slow onset. Diabetes Care 1995;18:1095-9. 
11. Urakami T, Suzuki J, Yoshida A, Saito H, Mugishima H. Incidence of children with slowly progressive form of type 1 diabetes detected by the urine glucose screening at schools in the Tokyo Metropolitan Area. Diabetes Res Clin Pract 2008;80:473-6.

12. Zimmet PZ, Tuomi T, Mackay IR, Rowley MJ, Knowles $\mathrm{W}$, Cohen $\mathrm{M}$, et al. Latent autoimmune diabetes mellitus in adults (LADA): the role of antibodies to glutamic acid decarboxylase in diagnosis and prediction of insulin dependency. Diabet Med 1994;11:299-303.

13. Naik RG, Brooks-Worrell BM, Palmer JP. Latent autoimmune diabetes in adults. J Clin Endocrinol Metab 2009;94:4635-44.

14. Urakami T, Kuwabara R, Habu M, Okuno M, Suzuki J, Takahashi S, et al. Clinical characteristics of non-obese children with type 2 diabetes mellitus without involvement of $\beta$-cell autoimmunity. Diabetes Res Clin Pract 2013;99:105-11.

15. Sugihara S, Sasaki N, Amemiya S, Kohno H, Tanaka T, Matsuura N. Analysis of weight at birth and at diagnosis of childhood-onset type 2 diabetes mellitus in Japan. Pediatr Diabetes 2008;9(4 Pt 1):285-90.

16. Morimoto S, Urakami T. Insulin resistance during puberty in non-obese Japanese children. Clin Pediatr Endocrinol 2011;20:1-6.

17. Urakami T, Habu M, Kuwabara R, Komiya K, Nagano N, Suzuki J, et al. Insulin resistance at diagnosis in Japanese children with type 2 diabetes mellitus. Pediatr Int 2012;54:516-9.

18. Yoon KH, Lee JH, Kim JW, Cho JH, Choi YH, Ko SH, et al. Epidemic obesity and type 2 diabetes in Asia. Lancet 2006;368:1681-8.

19. Chan JC, Malik V, Jia W, Kadowaki T, Yajnik CS, Yoon $\mathrm{KH}$, et al. Diabetes in Asia: epidemiology, risk factors, and pathophysiology. JAMA 2009;301:2129-40.

20. Tfayli H, Bacha F, Gungor N, Arslanian S. Phenotypic type 2 diabetes in obese youth: insulin sensitivity and secretion in islet cell antibody-negative versus -positive patients. Diabetes 2009;58:738-44.

21. Urakami T, Kuwabara R, Habu M, Yoshida A, Okuno M, Suzuki J, et al. Pharmacologic treatment strategies in children with type 2 diabetes mellitus. Clin Pediatr Endocrinol 2013;22:1-8.

22. Urakami T. New insights into the pharmacological treatment of pediatric patients with type 2 diabetes. Clin Pediatr Endocrinol 2018;27:1-8. 